\title{
Planned Dose
}

National Cancer Institute

\section{Source}

National Cancer Institute. Planned Dose. NCI Thesaurus. Code C82551.

The proposed dosage of the treatment. 